\title{
EDITORIAL
}

\section{0 volumes of Marine Ecology Progress Series (MEPS): a unique success story continues}

\author{
Otto Kinne* \\ Inter-Research, Nordbünte 23, 21385 Oldendorf/Luhe, Germany
}

In its 31st year, MEPS is publishing Vol. 400. The rise of MEPS, from small beginnings in 1979 to its establishment as the most highly ranked journal in its field, is unparalleled. The total number of articles published, representing the foremost marine ecological research, is now well over 10000.

MEPS has seen a variety of developments in the 4 years since the publication of Vol. 300:

- Yearly submissions have increased from about 900 to $>1100$, and the annual number of volumes published from 20 to 24 .

- The number of Contributing Editors (CEs) has increased from 23 to 49; the number of Review Editors, from 73 to 87 .

- The introduction of centralized manuscript handling via XPressTrack has increased efficiency, reduced the clerical workload of CEs, and helped the Editors-in-Chief to support the CEs' work.

- The Impact Factor has risen from 2.1 to 2.6. According to Eigenfactor.org, a non-profit project, MEPS is No. 1 by Eigenfactor, far ahead of all other journals, in the categories 'Marine \& Freshwater Biology' and 'Oceanography'.

- Our Open Access (OA) policy and the introduction of 'Feature Articles' (FAs) have further enhanced global visibility of the journal and provided a tangible reward for our best authors. According to our Web statistics, FAs are downloaded 5 times more frequently, and OA articles are downloaded 2.5 times more often than regular articles.
- Articles co-authored by CEs are now published with free OA.

- MEPS articles older than 5 years are available with OA, back to Vol. 1 in 1979.

As with other journals, referee selection and involvement have become more difficult in recent years; an increasing number of experts invited to review mss cannot afford the time required. Nevertheless, we are maintaining the high standard that MEPS is known for (3 to 4 reviews per manuscript).

Subscription numbers have remained robust, despite the current economic crisis and the resulting reduction in institutional budgets.

MEPS will not increase charges for OA, nor for color printing. No other fees will be introduced in the foreseeable future.

The rapid development of MEPS is complemented by the birth of 2 new sister journals: Aquatic Biology (AB), founded in 2007, and Aquaculture Environment Interactions (AEI), founded in 2009. AB has already published $>200$ articles. AEI will publish the first articles in 2010. Inter-Research now produces 8 journals.

The success of MEPS is largely due to: numerous high-quality contributions submitted, excellent performance of our editors and referees, the hard work and dedication of our technical and administrative staff, and last but not least, the loyalty of our subscribers.

Thank you all! 